now exist in the United States four types of large-scale computing machines: (1) the differential analyzer of the Massachusetts Institute of Technology, (2) an electromechanical computing device (the IBM Sequence Controlled Calculator) of the Harvard Computation Laboratory, (3) relay-type computing machines of the Bell Telephone Laboratories, and (4) an electronic computing machine (ENIAC) of the Ballistic Research Laboratory, Aberdeen Proving Ground, Md.

After the introductory article, the material in this Section falls under the general headings of Techinical Developments, Discussions, Bibliography, and News. The necessary editing is provided by the staff of the Machine Development Laboratory of the National Bureau of Standards. Correspondence regarding the Section should be directed to Dr. E. W. Cannon, 418 South Building, National Bureau of Standards, Washington 25, D. C.

\title{
THE ZUSE COMPUTER ${ }^{1}$
}

General Description. The Zuse computer is a relay-type digital machine. At present electro-magnetic telephone relays are used except in the storage unit, where special mechanical relays have been introduced. The machine operates upon numbers in dyadic representation and carries out automatically the ordinary algebraic operations, including division and extraction of square roots. Automatic handling of algebraic sign and of decimal point are provided. Numerical input is limited to the use of a manual keyboard, and output to a visual display panel. An automatically-sequenced program can be introduced by means of punched tape; however it must be followed through in a rigidly prescribed manner. ${ }^{2}$ Only directions as part of the program, and not numerical values, can be entered by means of the tape. The machine will handle numbers of five significant figures, within a range of magnitude of $10^{ \pm 20}$. Multiplying time is of the order of one second. Projected storage capacity is for 1024 numerical quantities, but only 16 cells have been constructed to date.

The machine is constructed of rather rough homemade components, so that it may be assumed that it occupies more space, and must operate more slowly, than would be necessary for a more carefully engineered model. The manual controls together with the visual display panel are built into a unit of approximately the shape and size of a small upright piano. The relay circuits are contained in four large cases roughly $6^{\prime} \times 3^{\prime} \times 1^{\prime}$. The storage component, with space provided for the projected 1024 cells, is contained in two boxes less than a yard square and $15^{\prime \prime}$ high. Zuse estimates about 1000 cells to the cubic yard. In addition to these parts there are an electric motor for operating the mechanical components, a drum commutator for operating the relays, a tape reader, and a tape punch.

Numerical Input and Output. The machine receives five-digit quantities in decimal notation. These are entered by successively pressing a combination of the 10 digit keys and the key for the decimal point. In addition, the decimal point may be shifted 6,12 , or 18 places to the right or left by pressing one of the keys $10^{+6}$ or $10^{-6}$ one, two or three times. The numbers so entered appear immediately in the form of illuminated lamps on the display panel. All numbers are entered thus in semilogarithmic decimal form and are automatically converted into dyadic representation. Twenty-two dyadic places are retained, which corresponds to something over six significant figures in decimal notation.

In operation all quantities are taken in dyadic semilogarithmic form with 
algebraic sign. Decimal point position and algebraic sign are handled automatically in all calculations.

Output is obtained by a visual display panel comprising seven columns of ten lamps each for the significant figures, with auxiliary lamps to indicate position of the decimal point, algebraic sign, and other special quantities. Any quantity in the machine may be called for on the display panel at any time, and is automatically converted into decimal notation before presentation.

No means of numerical input or output other than the keyboard and the display panel are provided.

Special Algebraic Devices. A special key is provided for zero, the reason being given that this quantity is unsuited for expression in semilogarithmic form. In addition to the special symbol for zero, a symbol, $\ll$, is provided on the display panel, signifying "of too small a magnitude for the scope of the machine." This would appear, for example, as the result of subtracting any number from itself. It is difficult to see the value of this arrangement, since it was admitted that the symbol " 0 ," for exactly zero, could never be obtained as the result of a computation.

Three expressions for infinity are employed. Of these, $+\infty$ and $-\infty$ indicate a result which is positive or negative but numerically beyond the scope of the machine; the third, $\pm \infty$, denotes a large quantity which is ambiguous as to sign, e.g., the result of dividing 1 by 0 or by $\ll$. An additional symbol, 0/0, signifies an indeterminate result, e.g., the result of dividing one very small quantity by another.

The algebraic combination of these various signs for infinite, infinitesimal, and indeterminate quantities is handled automatically in the same fashion as for ordinary numbers; no special attention is demanded of the operator, nor in preparation of an automatic program. Thus, for example, $(+\infty)-(+\infty)$ automatically yields $0 / 0$, etc.

One key is provided for the minus sign, - , and corresponding indicators for + and - on the display panel.

Likewise a key and indicator are provided for the imaginary unit, $i=\sqrt{ }-1$. However, operation with imaginary quantities is limited to the two rules that when the square root of a negative number is called for, the symbol $i$ will register, and that when two pure imaginaries are multiplied together a minus sign will register. Beyond this, the machine is not equipped for automatic computation with complex numbers.

Arithmetical Unit. The arithmetical unit contains two special storage cells. When numbers have been read into one or both of these cells, a key may be pressed directing that the two numbers be added, subtracted, multiplied or divided; or, in the case of a single number, that the negative, the square root, the double, the half, the 10 fold, the 10 th part of the first of these numbers be produced, or that the number be multiplied by $\pi$. Provision is planned for squaring, taking the reciprocal, and the maximum or minimum of two quantities, but these operations are not yet available. The result of an operation is in turn stored in the arithmetical unit. By pressing an appropriate key, the result of the operation can be made to appear upon the display panel as fast as it is computed.

The arithmetical operations are carried out by relay circuits. The conversion from decimal to dyadic representation is carried out by the relays 
as are used for addition, together with some auxiliary relays for controlling the operation. Information regarding the actual circuits employed was withheld; however, it was stated that the more complicated operations of division and extraction of square roots are carried through by the procedures used in hand computation.

Storage. The storage is planned to contain 1024 cells, each of which will hold a single numerical quantity with its algebraic sign and decimal position. For this purpose mechanical relays are used. These are of a special design, of which the details were not revealed. One layer of 16 cells is now in operation.

The relays appear to consist of a number of thin strips of metal lying between two plates of glass, not more than a quarter of an inch apart. Strips running in one direction through this layer represent the 16 cells, each containing one numerical quantity, while those in the perpendicular direction represent the individual digits, etc., of each cell.

Motion of the strips is controlled by electromagnetic relays that engage or disengage individual strips with an arm providing mechanical impulses at regular intervals. It would appear that motion of a particular strip representing a chosen cell exposes other moving parts, corresponding to each digit position in the cell, to the motion communicated by the various transverse digit. strips. The whole mechanism appeared quite compact and simple; however, detailed examination or description was denied.

There is no doubt that this type of storage is more compact than electromagnetic relays. One layer of 16 cells is roughly two feet square and $t^{\prime \prime}$ thick, to which must be added a few inches for the protruding ends and the operating mechanism. This storage was demonstrated and appeared satisfactory with regard to accuracy and speed of operation; however it was stated that some difficulty had resulted from the necessity of using lowquality metal.

In operation a number may be directed into any cell of the storage from either the input keyboard or the computer. The storage does not operate as an accumulator, and it is not necessary to clear a cell before use. If a second number is directed into a cell in which a number is already stored the first number will be completely lost and replaced by the second. Once a number is entered, it remains in the storage until replaced by another. A number in any cell can be read into the computer, or onto the display panel, at will and as often as is required.

Control Unit. The timing of the basic operations of the machine is controlled by an electric motor, which drives a cylindrical commutator operating the relays and also provides the mechanical drive for the storage relays, thus assuring synchronization. The actual speed of operation was stated to be about half what could ultimately be expected. This delay was attributed to difficulties with the electrical circuits rather than to the electromagnetic or mechanical relays themselves.

All operations and transfers are initiated by pressing a control key, and the completion of an operation is indicated by a special lamp on the display panel. Operating time was stated to be about one multiplication per second. This was confirmed in the demonstration. Extraction of a square root was timed at slightly over 5 seconds. Compared with these operating times, and 
with the time required for manual input and control, the time required for transfer from one part of the machine to another is negligible. It was stated that the mechanical relays had about the same speed of operation as the electromagnetic ones.

The timing of the sequence of arithmetical operations is controlled by the operator. When the signal flashes, indicating that one operation is complete, a key must be pressed to initiate the next operation. When the machine operates under automatic control, the completion of one operation causes the tape reader to advance and initiate the next.

Automatic Program. The machine may be operated by a punched film. This is ordinary celluloid moving picture film about an inch wide. An order may be punched on to the film in the form of some combination out of 8 holes, occupying two lines across the film. Numerical values cannot be entered by means of a tape, but only directions to transfer numbers between the storage and the computer, or to perform algebraic operations on the quantities in the computing unit. Before an automatic program can begin, all the initial values must be entered manually by way of the keyboard into the proper cells of the storage. After the calculation is complete, or at any time the operator chooses to intervene, the quantities in the storage may be read from the visual display panel.

The tape is prepared by directing the machine through the course of the calculation in exactly the same way, and possibly at the same time, as for non-automatic operation. The program can be of any length compatible with the storage capacity. An important limitation upon programming is that the machine must adhere to a prescribed linear course of operation. It cannot at any polnt choose between two subsequent programs on the basis of results already obtained; nor can it be directed to repeat automatically sub-programs within the same total program. A further incidental inconvenience is that even such constants as the number 1 must be entered into the storage along with the initial values, or else obtained as the result of trivial calculations. The speed of the machine for automatic operation is somewhat, but not a great deal, faster than for manual operation.

Present Status of Machine. There exists at present only one model of the Zuse machine. As has been indicated, this is incomplete both in mathematical and electrical design, and with regard to the engineering of the components. The machine is the property of Dipl. Ing. KonRaD ZUSE and is now housed in the cellar of a farm building in the village of Hopferau near Füssen, in southernmost Germany. Zuse has with him one assistant by the name of STUCKEN.

Improvements are being undertaken in the electrical circuits, as well as the completion of some of the projected algebraic operations which have not yet been installed, and the construction of more storage cells. This work is being carried out under rather primitive conditions and with inadequate material.

Zuse plans ultimately to convert the machine entirely to mechanical relays. On the basis of his experience with the mechanical relays now in use in the storage, and with earlier entirely mechanical machines, he is convinced that this will offer no serious difficulty once materials become available. It is his hope that after perfecting the design of a compact mechanical 
relay machine, he will be able to undertake commercial production in quantity.

So far as can be ascertained, Zuse is carrying out his work independently of any interest or assistance from outside sources.

Office of the Assistant Attaché

ROGER C. LYNDON

for Research, American Embassy,

London, England

1 This Report was prepared for the Office of Naval Research by Dr. Lyndon of the London branch office. The Zuse computer, invented by Konrad Zuse, is of interest in that it represents the work of German scientists in the field of large-scale computing devices. By 1943 Zuse had established his own firm for the construction of the computers he had invented. Two special-purpose aerodynamic machines developed during the war apparently proved successful. A general-purpose algebraic computer was also completed and had passed its test runs prior to the cessation of hostilities. Although these computers are interesting because of their similarity with wartime developments in the United States, it is clear that they are of limited utility in their present state of development. See also BIBLIOGRAPEY, Z-I, no. 3.

2 ZUSE states in a letter, received since the completion of this article, that it is now possible to enter constants by means of the punched tape; he also points out that with four tape readers and two tape punches, as called for in the design, the machine will be able to repeat programs and to choose between alternative sub-programs. He states further that when the designed wirings are complete, the machine will be fully equipped to handle problems involving complex quantities.

\section{TeChnical Developments}

\section{The Selectron-A Tube for Selective Electrostatic Storage (See frontispiece plate)}

We are engaged at the RCA Laboratories in the development of a storage tube for the inner memory of electronic digital computers. This work is a part of our collaboration with the Institute for Advanced Study in the development of a universal electronic computer. The present note describes briefly the principle of operation of the tube, which is still in its experimental stage. It is a summary of a paper presented at the "Symposium of Large Scale Calculating Machinery" at Harvard University on January 8, 1947; see $M T A C$, v. 2, p. 229-238.

The necessity of an inner memory in electronic digital computers has been realized by all designers. The high computing speed possible with electronic devices becomes useful only when sufficient intermediary results can be memorized rapidly to allow the automatic handling of long sequences of accurate computations which would be impractically lengthy by any other slower means. An ideal inner memory organ for a digital computer should be able to register in as short a writing time as possible any selected one of as many as possible on-off signals and be able to deliver unequivocally the result of this registration after an arbitrarily long or short storing time with the smallest possible delay following the reading call.

The selectron is a vacuum tube designed in an attempt to meet these ideal requirements. In it, the signals are represented by electrostatic charges forcefully stored on small areas of an insulating surface. The tube comprises an electron source which bombards the entire storing surface. The insulator can be a circular cylinder coaxial with a standard thermionic cathode. Between the cathode and the storing surface there are two orthogonal sets of 